\title{
Molecular methods in diagnostics of post-BCG vaccine adverse events
}

\author{
SYLWIA AGATA BRZEZIŃSKA ${ }^{1}$, TERESA BIELECKAㄹ, ANNA ZABOST, \\ AGNIESZKA GŁOGOWSKA ${ }^{l}$, MONIKA KOZIŃSKA ${ }^{l}$, EWA AUGUSTYNOWICZ-KOPEC ${ }^{l}$ \\ ${ }^{1}$ Department of Microbiology, National Tuberculosis and Lung Diseases Research Institute, Warsaw, Poland \\ ${ }^{2}$ Department of Pediatric Pulmonology and Allergy, Medical University of Warsaw, Warsaw, Poland
}

\begin{abstract}
Introduction: Bacillus Calmette-Guérin $(B C G)$ is the only tuberculosis vaccine available and although it has been routinely used for more than 80 years, its protective effect varies depending on the age and the form of tuberculosis. Due to the close analogy between the vaccine strain and other species of the Mycobacterium tuberculosis complex, molecular methods are recommended for differential diagnosis of post-BCG complications. The aim of the study was to assess usefulness of molecular methods in diagnosis of post-BCG vaccine adverse events (VAEs).

Material and methods: M. tuberculosis complex strains obtained in 2011-2017 from 68 ill children were subjected to molecular analysis.

Results: Molecular analysis of 68 strains showed $100 \%$ agreement between the results in the GenoType MTBC method and the multiplex PCR method. For the strains isolated from 45 patients with suspected VAE, M. bovis BCG was obtained, whereas the strains isolated from the remaining 23 children were identified as M. tuberculosis. The analysis confirmed the close relationship between the result of identification and the type of material as well as the patient's age.

Conclusions: The use of genetic methods enables quick and detailed diagnostics of infections caused by $M$. bovis BCG, which allows for the confirmation or exclusion of VAE.
\end{abstract}

Key words: Bacillus Calmette-Guerin, Mycobacterium tuberculosis, vaccines.

(Cent Eur J Immunol 2020; 45 (2): 130-135)

\section{Introduction}

Tuberculosis in children remains a health problem in many countries, both developed and developing, and it is closely related to the disease prevalence in adults, which is intensifying in many regions of the world. Accurate data on the number of children with tuberculosis are not precisely known. According to the WHO Global Tuberculosis Report, in 2017, children accounted for $7.1 \%$ of tuberculosis reported cases [1].

Since the introduction of the tuberculosis vaccine in the 1920s, it has been the subject of research and discussions on its effectiveness. The protective effect of the vaccine is variable (from approx. $40 \%$ to $80 \%$ ), depending on the age and the form of the disease. Its highest effectiveness has been demonstrated in the prevention of severe forms of tuberculosis in children, especially meningitis (60-80\% of protection), while the lowest in relation to the widespread of tuberculosis in adults [2].

The original, attenuated Bacillus Calmette-Guérin (BCG) strain regarded worldwide as a tuberculosis vaccine since 1921, under the influence of specific and ever-changing production conditions has undergone many modifications. As a result, $49 \mathrm{BCG}$ sub-strains were created. One of them is the Mycobacterium bovis BCG Moreau sub-strain included in the vaccine commonly used in Poland since 1952 [3].

The International Union Against Tuberculosis and Lung Disease has developed guidelines regarding the criteria for the cessation of BCG vaccination in the population. The main indication is the decline in the incidence of pulmonary tuberculosis with positive sputum smears less than $5 / 100,000$ over the last three years. In Poland, in 2016, the average ratio was $6.8 / 100,000$ and showed territorial differentiation with the highest ratio in Śląskie 8.4/100,000 and the lowest in Lubuskie Voivodship 4.0/100,000 [4]. In countries with low prevalence of tuberculosis, the criteria to cease common BCG vaccination are the following: number of cases of tuberculous meningitis (TBM) in children under 5 years of age, less than 1 case per 10 million people in the general population, persisting for the last 5 years. In

Correspondence: Sylwia Agata Brzezińska, MSc, Department of Microbiology, National Tuberculosis and Lung Diseases Research Institute, 26 Płocka St., 01-138 Warsaw, Poland, e-mail: s-brzezinska@wp.pl Submitted: 26.03.2019; Accepted: 30.04.2019 
Poland, between 2012-2016, in children under 5 years of age, TBM was diagnosed twice. Another criterion concerns the average annual risk of tuberculosis infection (Styblo coefficient), which must not exceed $0.1 \%$. In Poland, in 2016, this was $0.14 \%$. In addition to the above-mentioned indicators, if discontinuation of BCG vaccination is considered an analysis of the occurrence of serious complications after BCG vaccination and its cost-effectiveness should also be performed. Moreover, preservation of vaccination should be considered in some well-defined risk groups for tuberculosis infection [5]. The BCG vaccine is one of the safest live bacterial vaccines; however, the immunization process can cause vaccine adverse events (VAEs), such as skin lesions at the site of injection, enlargement of local lymph nodes, or osteoarthritis. The most serious complication following BCG vaccination is severe generalized infection with mycobacterium strain, which usually occurs in patients with immunologic deficiency, such as severe combined immunodeficiency, IFN- $\gamma$ or Il-12 receptor defect, or chronic granulomatous disease [6]. Radiological reports on the disseminated BCG infection mostly describe changes, which indicate osteomyelitis [7].

In Poland, vaccination takes place on the first day after birth; it is important to collect the family history of congenital immunodeficiency. The vaccination should be postponed until the exclusion of immunodeficiency in the case of suspected immune defect in the neonate.

Epidemiology of complications, specific for the BCG vaccine, varies from country to country. Although these reactions are rare, they range from mild local changes to a dissemination of potentially deadly disease. The etiology of complications may be related to the vaccine itself (technical errors, strain used) or the patient's condition. The latter reason occurs in cases of disseminated M. bovis BCG infection, concurrent with primary or acquired immunodeficiency.

Since every unwanted post-vaccination reaction is defined as a "medical symptom temporarily associated with the vaccination, which may have multiple causes", the incidence of $M$. bovis BCG infection is not precisely known. The vaccine consists of live attenuated bacilli; therefore, children with immunologic deficiency may experience generalized $M$. bovis BCG infection. In patients with a properly functioning immune system, these complications are extremely rare. However, the risk of disseminated BCG disease increases several hundred times in infants infected with HIV, compared to the risk in uninfected infants. Therefore, BCG vaccination is contraindicated in infants born to HIV-positive mothers until the infection in a child is ruled out [8].

The diagnosis of $M$. bovis BCG infection is based on physical examination and the results of microbiological tests. The use of conventional methods for identification of mycobacteria does not allow to distinguish the BCG strain from other Mycobacterium tuberculosis complex spe- cies, which include $M$. tuberculosis, M. canetti, M. bovis, M. bovis BCG, M. caprae, M. africanum, M. microti, and M. pinnnipedi. The identification of $M$. bovis BCG as etiological factor of the disease is especially important due to the different treatment scheme. Local skin and nodal reactions occurring post-BCG vaccination usually do not need a therapy, while cutaneous or nodal tuberculosis must be treated. Moreover, in case of $M$. bovis BCG dissemination, urgent diagnostics of immunodeficiency should be implemented. For this reason, some VAE cases are classified as M. tuberculosis complex (MTBC) using microbiological tests but were registered and treated as tuberculosis.

Identification of the $M$. bovis BCG species can be performed only in a few highly specialized laboratories. An absolute indication for the above is the clinical suspicion of VAE. Therefore, in the case of strains cultured from children, it is recommended to perform a detailed diagnosis that differentiates species within the MTBC.

Commercial methods used for the routine genetic diagnostics also do not distinguish between species within the above-mentioned complex. M. bovis is characterized, such as other complexes, by a long growth time (2-6 weeks) and resistance to acid in Z-N staining. Due to the close analogy of morphological, physiological, and biochemical features, specific molecular diagnostics are recommended for differential diagnosis. The current state of knowledge is that a mutation associated with the loss of virulence of the M. bovis BCG vaccine strain is a deletion in the RD1 region. This deletion differentiates $M$. bovis BCG from other species belonging to the $\mathrm{MTBC}$, in which this sequence occurs [9].

Adverse reactions following BCG vaccination are divided into local, nodal, and distant reactions. Local complications (abscess or ulceration) and local lymph nodes involvement (inflamed and/or enlarged nodes), usually do not require medical intervention but only protection against superinfection. In the case of node suppuration and the tendency to create a node-cutaneous fistula, punctures with evacuation of purulent content significantly reduce the healing time of changes [10]. In Poland, the above-mentioned complications are among the most common reactions following BCG vaccination, and may occur in healthy children without immune system function impaired. Long-term complications, such as osteoarthritis or generalized BCG mycobacteria dissemination, are serious and require detailed diagnostics of the patient and combined treatment. The Moreau Brazilian sub-strain commonly used in Poland belongs to BCG sub-strains with lower reactogenicity compared to the Danish or French sub-strains. This is associated with a smaller number of VAEs and their milder course. The occurrence of distant complications after vaccination with the Moreau sub-strain indicates the coexistence of immunologic deficiency, requiring detailed diagnostics in the immunology ward [10]. 
In view of the non-characteristic initial symptoms of BCG dissemination, the possibility of bacteriological confirmation of the disease etiology, directs and facilitates further diagnostic as well as therapeutic procedures. The prognosis largely depends on the state of immune system, the type of immune disorder, and its treatment options as well as the antimycobacterial therapy used. Although therapeutic protocols are still controversial, there is an increasing number of reports on the guidelines for diagnosis and treatment of complications following BCG-vaccination [11].

The aim of the study was to assess usefulness of molecular methods in diagnosis of post-BCG VAEs.

\section{Material and methods}

The MTBC strains obtained between 2011 and 2017 from 68 ill children with suspected VAE were subjected to the molecular analysis (Table 1). The isolated DNA was examined with the use of two genetic methods differentiating species within the MTBC.

GenoType MTBC test (Hain Lifesciencehttps://www. hain-lifescience.de/en/) is a qualitative test, which uses DNA-strip technology, differentiating species within the MTBC. The procedure consists of the following steps: mycobacteria's DNA isolation from the obtained culture, amplification, and reverse hybridization. The presence of bands at control sites (CC, UC, and MTBC) indicates the correct course of the reaction and the presence of mycobacteria genetic material.

PCR multiplex [12] is a test to identify M. bovis BCG, which detects the RD1 deletion fragment that is characteristic for this species. The strains involved in MTBC have the RD1 sequence, with $9.5 \mathrm{~kb}$ in size, with the exception of the M. bovis BCG strain, whose genome does not contain this sequence.

Table 1. Clinical materials from children with suspected vaccine adverse events

\begin{tabular}{lc}
\hline $\begin{array}{l}\text { The type of material from } \\
\text { which the strain was obtained }\end{array}$ & $\begin{array}{c}\text { Number } \\
\text { of materials }\end{array}$ \\
\hline Biopsy from the site of vaccination & 24 \\
\hline Lymph node & 13 \\
\hline Gastric lavage & 12 \\
\hline Swab from abscess & 4 \\
\hline Bronchoaspirates & 4 \\
\hline Bronchial lavage & 5 \\
\hline Sputum & 2 \\
\hline Pleural fluid & 2 \\
\hline Swab from fistula & 1 \\
\hline Bursa fluid & 1 \\
\hline
\end{tabular}

The significance of differences in means of different groups was determined by using Student's unpaired $t$-test. The differences were considered significant when a $p$ value of $<0.05$ was obtained.

\section{Results}

Molecular analysis of 68 strains showed $100 \%$ agreement between the results obtained in the GenoType MTBC method and the multiplex PCR method. In both tests, for the strains isolated from 45 patients with suspected VAE, M. bovis BCG was obtained, while the strains isolated from the remaining 23 children were identified as $M$. $t u$ berculosis. In the analysis, a close relationship was found between the type of material and the result obtained.

The 45 strains identified as M. bovis BCG were dominated by cultures obtained from materials originating from the vicinity of the site of vaccination. These were biopsies or swabs from ulcers, purulent lesions, and subcutaneous abscesses, which accounted for a total of $64 \%$. A large group was also represented by materials originating from regional (axillary, supraclavicular, subclavicular) lymph nodes (up to $29 \%$ ). The remaining $7 \%$ of the strains were obtained from clinical materials, not related to the place of vaccination, i.e. gastric lavage and bursa fluid. The obtained result of identification was closely related to the age of patients. The patients with confirmed presence of the M. bovis BCG species, predominantly (93\%) represented the age range from 2 months to 4 years of age. Only seven percent of patients with $M$. bovis BCG were over 10 years of age. The group of patients with confirmed presence of M. tuberculosis represented the age range from 4 to 14 years of age (Table 2).

Among 23 analyzed patients for whom M. tuberculosis results were obtained, all cultures came from clinical materials characteristic of pulmonary tuberculosis: gastric lavage (approx. 48\%), bronchial lavage (approx. 22\%), bronchoaspirates (approx. 13\%), and pleural fluid and sputum (approx. 9\%) (Table 3). For the analyzed groups, the results presented no statistically significant differences (Tables 2 and 3).

For the analyzed strains, drug resistance tests were performed on four basic drugs, with pyrazinamide, among others. All the strains were sensitive to RMP, INH, and EMB. The resistance to PZA was $100 \%$ characteristic of the strains identified as $M$. bovis BCG. This is in accordance with the resistance to pyrazinamide, which is natural for this species.

\section{Discussion}

In 2015, the World Health Organization (WHO) announced its "End TB strategy", the aim of which was to reduce the number of TB deaths by $95 \%$ until 2035, the decrease in the number of patients with TB by $90 \%$, and the 
Table 2. Numbers of M. bovis BCG and M. tuberculosis in patients at different ages

\begin{tabular}{lccccc}
\hline Age & $\begin{array}{c}\text { M. bovis } \\
\text { BCG }\end{array}$ & Percent & $\begin{array}{c}M . \\
\text { tuberculosis }\end{array}$ & Percent & $\boldsymbol{p}$-value \\
\hline 0-6 months & 20 & 44.4 & 0 & 0.0 & 0.7 \\
\hline 6-12 months & 17 & 37.8 & 0 & 0.0 & 0.7 \\
\hline 1-2 years & 3 & 6.7 & 0 & 0.0 & 0.9 \\
\hline 2-3 years & 1 & 2.2 & 0 & 0.0 & $>0.9$ \\
\hline 3-4 years & 1 & 2.2 & 0 & 0.0 & $>0.9$ \\
\hline 4-6 years & 0 & 0.0 & 2 & 8.7 & 0.9 \\
\hline 6-8 years & 0 & 0.0 & 3 & 13.0 & 0.9 \\
\hline 8-10 years & 0 & 0.0 & 5 & 21.7 & 0.8 \\
\hline > 10 years & 3 & 6.7 & 13 & 56.5 & 0.7 \\
\hline
\end{tabular}

incidence of 10 cases per 100,000 inhabitants. To achieve these goals, the WHO predicted an annual fall in tuberculosis incidence by $10 \%$ until 2025 and $17 \%$ from 2025 till 2035. However, according to the latest WHO reports, the number of estimated TB cases in the world has increased by $20 \%$, from 8.6 million in 2012 to 10.4 million in 2016 and 10 million in 2017. These epidemiological data clearly indicate that tuberculosis will not be eradicated in the near future and will continue to be among the top ten causes of death worldwide $[1,13,14]$.

Therefore, the WHO continues to recommend vaccination of infants in endemic countries and in high-risk groups of tuberculosis infection. In 2017, 158 countries reported the presence of BCG vaccination in the Protective Vaccination Program. For the majority, as many as 120 of them, the vaccination coverage levels were above $90 \%$ [1].

For many years, research has been carried out on mortality of children vaccinated with $M$. bovis BCG. Since tuberculosis vaccination is performed in the neonatal period, it provides early protection, therefore restricting the critical period of sensitivity. It has also been suggested that vaccination at such an early stage may have an immunomodulatory effect, increasing the early resistance to viral and bacterial pathogens, resulting in the possibility of limiting the number of doses. Vaccinations of newborns are also easy to implement, as they take place immediately after delivery in hospital conditions, i.e. at the key point of contact with healthcare systems. Thus, neonatal vaccines achieve high vaccination coverage levels within the population, which is particularly important in the areas with limited access to healthcare [15]. In Sweden, shortly after the introduction of vaccination in 1920, lower mortality rates were recorded in children vaccinated against tuberculosis, as compared to the group of unvaccinated children. It was found that the reduction in mortality was most likely because of the influence of the vaccine on specific immunity [16]. A similar thesis was also presented in En-
Table 3. Numbers of $M$. bovis BCG and M. tuberculosis from different clinical samples

\begin{tabular}{lccccc}
\hline Materials & $\begin{array}{c}\text { M. bovis } \\
\text { BCG }\end{array}$ & Percent & $\begin{array}{c}\text { M. } \\
\text { tuberculosis }\end{array}$ & Percent & $p$-value \\
\hline $\begin{array}{l}\text { From the site } \\
\text { of injection } \\
\text { or regional } \\
\text { lymph nodes }\end{array}$ & 42 & 93.3 & 0 & 0 & 0.5 \\
\hline Other & 3 & 6.7 & 23 & 100 & 0.7 \\
\hline
\end{tabular}

gland, according to the data from the 1950s, a decrease in mortality among vaccinated children was observed in relation to the unvaccinated population [17]. There have also been numerous reports suggesting non-specific effects of BCG vaccination. Jesper Kjaergaard in his work refers to a research confirming the correlation between vaccination and prevention of atopic dermatitis, efficacy in treating melanoma, multiple sclerosis (MS) or type 1 diabetes. The rationale for this thesis is that $\mathrm{BCG}$ has a regulatory effect on the immune system to a certain extent, which in turn may have an impact on the child's growth and psychomotor development [18]. However, a close relationship between the safety of BCG vaccination and the state of the child's immune system should be considered. This dependence is emphasized in the work by Roglić et al., describing the case of an 8-month-old patient who was diagnosed with HIV infection and disseminated BCG disease [19]. In this child, ulceration at the place of vaccination was observed together with enlargement of ax-

Table 4. Vaccine adverse event (VAE) cases of registered BCG post-vaccination reactions in 2017 [21]

\begin{tabular}{lc}
\hline The nature of the adverse VAE reaction & Number \\
\hline $\begin{array}{l}\text { Purulent pustules with a diameter of }>1 \mathrm{~cm} \\
\text { in a newborn or }>2 \mathrm{~cm} \text { in older children }\end{array}$ & 126 \\
\hline
\end{tabular}

Ulceration with a diameter of $>1 \mathrm{~cm}$ in a newborn 19 or $>2 \mathrm{~cm}$ in older children

Enlargement of the surrounding lymph nodes $>1 \mathrm{~cm}$ :

\begin{tabular}{ll}
\hline Total & 291 \\
\hline Including festering nodes - total/with fistula & $36 / 8$ \\
\hline Subcutaneous abscess: & 127 \\
\hline Total & 19 \\
\hline With fistula & 5 \\
\hline Lymph node type calcium serum necrosis & 5 \\
\hline Generalized BCG mycobacterium infection & 1 \\
\hline Abortive Koch phenomenon & - \\
\hline Nodular erythema & 2 \\
\hline Keloid (keloidal scar) & 3 \\
\hline Allergic reaction &
\end{tabular}


illary lymph nodes, followed by generalized symptoms of infection. Mycobacteria were cultured from the site of skin sores, which were then identified as M. bovis BCG. Therefore, it was confirmed that in a patient with HIV, there was a disseminated infection of $M$. bovis BCG. The case study indicates that coexisting HIV infection may result in an adverse post-vaccination reaction in the form of a life-threatening disseminated M. bovis BCG infection. Hence, it seems reasonable to include pregnant women in a screening program for HIV infection. This will allow determination of the HIV status for the mother and newborn, and in the case of a positive result - abandoning the BCG vaccination [19].

The form of a generalized $M$. bovis BCG infection is a rare reaction. In Poland, in 2017, 5 cases were reported, which constituted about $0.8 \%$ of all registered VAEs following $M$. bovis BCG injection.

Misery and Combemale in their research presented rare forms of VAE post-BCG vaccinations, including lupus vulgaris and urticarial vasculitis. These are examples of extremely rare reactions just like the form of generalized infection, conditioned by the co-existence of certain predispositions [20]. The most frequently VAEs reported in literature are reactions occurring directly at the site of vaccination. In Poland, in 2017, they constituted about $98 \%$ of registered BCG post-vaccination reactions (Table 4) [21].

A similar tendency was observed in our research. The overwhelming majority, as many as $93 \%$ of the cultures identified as M. bovis BCG, came from clinical materials taken directly from the injection site or the nearby lymph nodes.

The BCG vaccine first used in the 1920s remains the only vaccine against tuberculosis. Despite its widespread use, the slow decline in morbidity emphasizes the need for a much more effective vaccine, providing protection against all forms of tuberculosis in all age groups. In parallel with the research on new vaccines, the studies which aim to better understand the pathogenesis of mycobacteria and the mechanisms of immune response during infection have been conducted. The results of these studies are of key importance in assessing the effectiveness of the new generation of tuberculosis vaccines in the preclinical and clinical studies. At present, clinical trials are being carried out for 12 vaccines. The most advanced study concerns Vaccae ${ }^{\mathrm{TM}}$ vaccine, which entered the third phase of research, in attempts to evaluate the efficacy and safety in the prevention of tuberculosis in high-risk groups. These are the largest studies on tuberculosis vaccine taken over the last decade involving 10,000 people aged $15-65$, with a positive PPD test [1]. The main goal of all initiatives undertaken in this field is the development of a new vaccine, which is characterized by maximum effectiveness, while maintaining a minimal risk of adverse post-vaccination reactions.

\section{Conclusions}

The analysis of materials from children with suspected VAEs reveals the need for detailed diagnostic tests that differentiate $M$. bovis BCG from other MTBC species. The modern molecular methods enable confirmation or exclusion of VAEs in children with suspected tuberculosis.

\section{The authors declare no conflict of interest.}

\section{References}

1. World Health Organization (2018): Global tuberculosis report 2018. Geneva.

2. Kjaergaard J (2016): Bacillus Calmette-Guérin vaccination at birth:Effects on early childhood infections, growth, and development. Dan Med J 63: B5304.

3. Corbel MJ, Fruth U, Griffiths EI, et al. (2004): Report on a WHO consultation on the characterisation of BCG strains. Imperial College, London 15-16 December 2003.Vaccine 22: 2675-2680.

4. Korzeniewska-Koseła M (ed.) (2017): Gruźlica i choroby układu oddechowego w Polsce w 2016 roku. Instytut Gruźlicy i Chorób Płuc, Warszawa.

5. [No authors listed] (1994): Criteria for discontinuation of vaccination programmes using Bacille Calmette-Guerin (BCG) in countries with a low prevalence of tuberculosis. A statement of the International Union Against Tuberculosis and Lung Disease. Tuber Lung Dis 75: 179-180.

6. Bernatowska E, Wolska-Kuśnierz B, Pac M, et al. (2007): Risk of BCG infection in primary immunodeficiency children. Proposal of diagnostics, prophylactic and therapeutic guidelines for disseminated BCG based on experience In the Department of Immunology, Childrens Memorial Health Institute In Warsaw between 1980-2006. Centr Eur J Immunol 32: 221-225.

7. Han TII, Kim I-O, Kim WS, et al. (2000): Disseminated BCG infection in a patient with severe combined immunodeficiency. Korean J Radiol 1: 114-117.

8. Bielecka T, Augustynowicz-Kopeć E, Gonerko P, et al. (2018): Recommendations for the management of tuberculosis in children. KOMPASS TB. Part 1: Tuberculosis prevention. Adv Respir Med 86: 149-157.

9. Krysztopa-Grzybowska K, Lutyńska A (2016): Microevolution of BCG substrains. Postepy Hig Med Dosw 70: 12591266.

10. Komorowska-Piotrowska A, Bielecka T, Feleszko W (2015): Rozpoznawanie i leczenie niepożądanych odczynów po szczepieniu BCG. Terapia 2: 62-65.

11. Kourime M, Akpalu ENK, Ouair H, et al. (2016): BCGitis/ BCGosis in children: Diagnosis, classification and exploration. Arch Pediatr 23: 754-759.

12. Talbot EA, Wiliams DL, Frothingham R (1997): PCR identification of Mycobacterium bovis BCG. J. Clin Microbiol 35: 566-569.

13. Dheda K, Barry CE, Martens G (2015): Tuberculosis. Lancet 6736: 1-17.

14. Dudnyk A, Chesov D, Lange Ch (2018): Mission impossible: the End TB strategy. Int J Tuberc Lung Dis 22: 121-122. 
15. Saso A, Kampmann B (2017): Vaccine responses in newborns. Semin Immunopathol 39: 627-642.

16. Naeslund C (1931): Experience de vaccination par le BCG dans la provine du norbotten. Rev Tuberc 12: 617-636.

17. Hart PD, Sutherland I (1977): BCG and vole Bacillus vaccines in the prevention of tuberculosis in adolescence and early adult life. Br Med J 2: 293-295.

18. Kjaergaard J (2016): Bacillus Calmette-Guérin vaccination at birth: Effects on early childhood infections, growth, and development. Dan Med J 63: B5304.

19. Roglić S, Dickson D, Miše B, et al. (2016): Successful Treatment of Disseminated Bacillus Calmette-Guérin Disease in an HIV-Infected Child with a linezolid- containing regimen. Case Rep Infect Dis 2016: 1528981.

20. Misery L, Combemale P (1993): BCG-vaccine-induced lupus vulgaris and urticarial vasculitis. Dermatology 186: 274.

21. NIZP-PZH Zakład Epidemiologii Chorób Zakaźnych i Nadzoru (2018): Vaccinations in Poland in 2017. 